\title{
The interaction between stray electrostatic fields and a charged free-falling test mass
}

\author{
F. Antonucci, ${ }^{1}$ A. Cavalleri, ${ }^{2}$ R. Dolesi,${ }^{1}$ M. Hueller, ${ }^{1}$ D. Nicolodi, ${ }^{1}$ H. B. Tu, ${ }^{1}$ S. Vitale,${ }^{1}$ and W. J. Weber ${ }^{1}$ \\ ${ }^{1}$ Dipartimento di Fisica, Università di Trento, and I.N.F.N., Gruppo di Trento, 38123 Povo (TN), Italy \\ ${ }^{2}$ Istituto di Fotonica e Nanotecnologie, C.N.R.- Fondazione Bruno Kessler, 38123 Povo (TN), Italy
}

(Dated: March 2, 2022)

\begin{abstract}
We present an experimental analysis of force noise caused by stray electrostatic fields acting on a charged test mass inside a conducting enclosure, a key problem for precise gravitational experiments. Measurement of the average field that couples to test mass charge, and its fluctuations, is performed with two independent torsion pendulum techniques, including direct measurement of the forces caused by a change in electrostatic charge. We analyze the problem with an improved electrostatic model that, coupled with the experimental data, also indicates how to correctly measure and null the stray field that interacts with test mass charge. Our measurements allow a conservative upper limit on acceleration noise, of $2 \mathrm{fm} / \mathrm{s}^{2} / \mathrm{Hz}^{1 / 2}$ for frequencies above $0.1 \mathrm{mHz}$, for the interaction between stray fields and charge in the LISA gravitational wave mission.
\end{abstract}

PACS numbers: 04.80.Nn, 07.87.+v, 91.10.Pp, 41.20.Cv

Limiting stray forces on a test mass (TM) is crucial for precise experimental gravitation, from gravitational wave $(\mathrm{GW})$ observation $[1-3]$ to tests of the equivalence principle [4], short range gravity [5, [6], and relativistic gyroscope precession [7, 8]. In all these experiments, electrostatic force noise is cited as a precision-limiting effect.

The orbiting GW observatory LISA (Laser Interferometry Space Antenna [3]) requires, along its sensitive $x$ axis, free-fall to within $3 \mathrm{fm} / \mathrm{s}^{2} / \mathrm{Hz}^{1 / 2}$ residual acceleration $-6 \mathrm{fN} / \mathrm{Hz}^{1 / 2}$ force noise - at frequencies $0.1-3 \mathrm{mHz}$. For LISA and its precursor LISA Pathfinder [9, 10], the TM is a $46 \mathrm{~mm}$ gold-coated cube, inside a co-orbiting satellite and shielded, without mechanical contact, by the gold-coated surfaces of a capacitive position sensor [11, 12], which can also apply actuation voltages.

While the sensor is nominally an equipotential shield, two factors can produce electrostatic forces relevant at the fN-level. First, the floating TM accumulates charge from cosmic and solar particles, with an expected net rate of order $50 \mathrm{e} / \mathrm{s}$ [13]. Second, real metals display stray potential differences [14, 15] between different points on a single conducting surface. These arise in different exposed crystalline facets and surface contamination. Typical observed average potential differences between roughly centimeter-size regions of a gold surface are of order $10-100 \mathrm{mV}$ [16-19].

By itself, TM charge $q$ creates a force gradient coupling to spacecraft motion, requiring periodic discharge [20]. Stray potentials $\delta V$ also create force gradients, whose $d^{-3}$ - or stronger 21] - dependence on the TM - sensor gap $d$, motivates large, several millimeter, gaps for LISA. Fluctuations in $\delta V$ also create force noise.

The mutual interaction between charge and stray potentials 22 25] can be written, to linear order in $q$,

$$
F_{x}=-\frac{q}{C_{T}}\left|\frac{\partial C_{X}}{\partial x}\right| \Delta_{x} .
$$

$\Delta_{x}$ is an effective potential difference proportional to $\frac{\partial F_{x}}{\partial q}$ and will be calculated shortly. $C_{X}$ and $C_{T}$ are, respectively, the TM capacitances to an $X$-electrode and the entire sensor (see Fig. 1).

This interaction produces force noise in two ways. First, any residual $\Delta_{x}$ multiplies random charge noise,

$$
\begin{aligned}
S_{F(\delta q)}^{1 / 2} & =\frac{S_{q}^{1 / 2}}{C_{T}}\left|\frac{\partial C_{X}}{\partial x}\right| \Delta_{x} \\
& \approx 7 \mathrm{fN} / \mathrm{Hz}^{1 / 2} \times\left(\frac{\Delta_{x}}{0.1 \mathrm{~V}}\right)\left(\frac{\lambda_{e f f}}{300 / \mathrm{s}}\right)^{1 / 2}\left(\frac{10^{-4} \mathrm{~Hz}}{f}\right) .
\end{aligned}
$$

$\Delta_{x} \approx 100 \mathrm{mV}$ is typical for LISA prototype sensors 16 , 17, [26]. $\lambda_{\text {eff }}$ is the equivalent single charge event rate that gives a "red" Poissonian shot noise $S_{q}=\frac{2 e^{2} \lambda_{e f f}}{\omega^{2}}$, estimated at roughly $300 / \mathrm{s}$ [13], larger during solar flare events [27]. This random charge force noise $S_{F(\delta q)}$ can be eliminated by nulling $\Delta_{x}$ with applied voltages [16, 25].

Second, fluctuations in $\Delta_{x}$ will multiply any nonzero TM charge to produce force noise,

$$
\begin{aligned}
S_{F\left(\delta \Delta_{x}\right)}^{1 / 2} & =\frac{q}{C_{T}}\left|\frac{\partial C_{X}}{\partial x}\right| S_{\Delta_{x}}^{1 / 2} \\
& \approx 1.3 \mathrm{fN} / \mathrm{Hz}^{1 / 2} \times\left(\frac{q}{10^{7} e}\right)\left(\frac{S_{\Delta_{x}}^{1 / 2}}{100 \mu \mathrm{V} / \mathrm{Hz}^{1 / 2}}\right) .
\end{aligned}
$$

$10^{7} e$ is roughly two days of accumulated charge and a reasonable discharge threshold.

This Letter addresses these two sides of the $q-\delta V$ interaction. Our analysis, considering spatial surface potential variations on both the TM and sensor, highlights systematic errors in measuring $\Delta_{x}$ with applied electrostatic fields, consistent with our experimental data. Force noise from field fluctuations is then addressed by measurements of stray potential fluctuations. Experiments employ a hollow LISA-like TM suspended as a torsion pendulum inside a prototype LISA capacitive sensor connected to a prototype sensing and actuation electronics [16]. All relevant surfaces have been sputtered with gold and held 

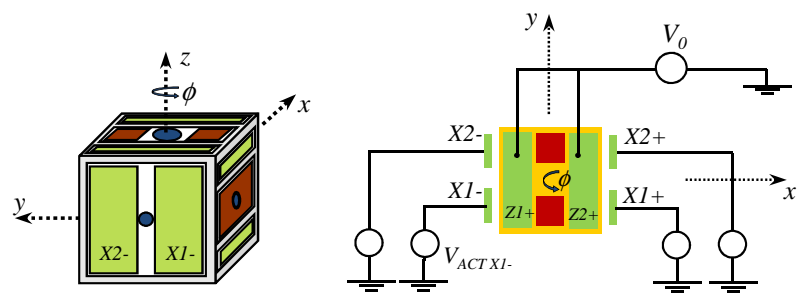

FIG. 1. Capacitive sensor, including (right) $X$ and $Z$ electrode connections in the $Z$-modulation experiments. The TM - electrode gaps are $4,2.9$, and $3.5 \mathrm{~mm}$ on, respectively, the $X, Y$, and $Z$ faces. The presented torsion pendulum measurements detect the rotation $\phi$.

under vacuum for more than a year. Our measurements in this flight-realistic configuration allow a conservative upper limit for the TM acceleration noise caused by the interaction between charge and stray fields.

The electrostatic interaction is modeled as a patchwork of discrete TM and sensor (S) surface domains at potentials $V_{i}$ (see Fig. 21), coupled by capacitors $C_{i j}$, with $q_{i}=\sum_{j} C_{i j}\left(V_{i}-V_{j}\right)[28]$. Stray potentials are defined by ideal generators $\delta V_{i}$. For a sensor domain, $V_{i}=\delta V_{i}$ or, if located on an electrode attached to generator $V_{A C T n}$, $V_{i}=\delta V_{i}+V_{A C T n}$. For a TM domain, $V_{i}=\delta V_{i}+V_{T M}$, where $V_{T M}$ is an effective average TM potential [29],

$$
V_{T M}=\frac{q}{C_{T}}+\frac{\sum_{j(S)} C_{S j} V_{j}}{C_{T}}
$$

where index $j(S)$ restricts the sum to sensor domains. $C_{S j} \equiv \sum_{i(T M)} C_{i j}$ is the total capacitance between sensor domain $j$ and all TM domains, and $C_{T} \equiv \sum_{j(S)} C_{S j}$ is the total TM capacitance to the sensor.

The force on the TM along the $x$ axis is

$$
F_{x}=\frac{1}{2} \sum_{i, j<i} \frac{\partial C_{i j}}{\partial x}\left(V_{i}-V_{j}\right)^{2},
$$

summing over all domain pair capacitances, including those with nearby domains on both the TM or sensor [30]. This combines with Eqn. 4 to yield the charge de-

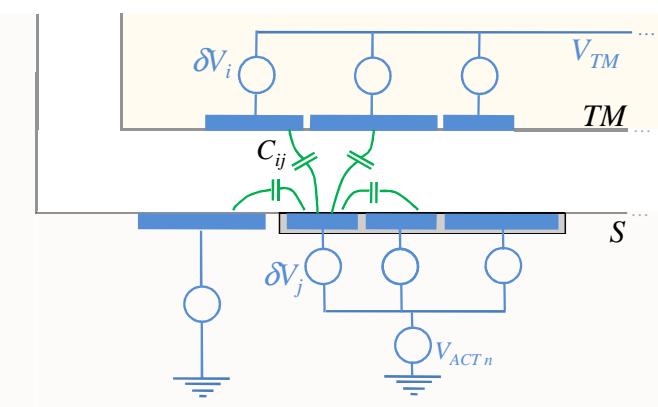

FIG. 2. Schematic of the electrostatic model, with capacitively coupled sensor (S) and TM domains. pendent force. For a centered TM, such that $\frac{\partial C_{T}}{\partial x}=0$,

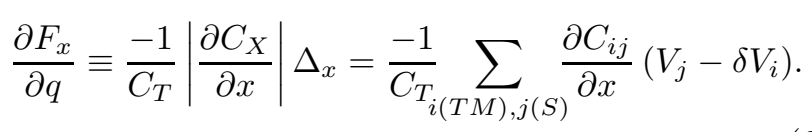

The derivative $\frac{\partial C_{X}}{\partial x}$ normalizes $\Delta_{x}$ to a single $X$ electrode potential, such that $+V$ applied to electrode $X 1+$ or $X 2+$ increases $\Delta_{x}$ by $V$, with the opposite change obtained with $X 1-$ or $X 2-$. Equation 6 differs from the analogous formula in Ref. [25] as it includes the spatially varying TM potential.

Shear forces arise naturally in Eqn. 5, in contrast with equipotential TM models 20, 25], for which the relevant derivative in Eqn. 5 becomes $\frac{\partial C_{S j}}{\partial x}$. Nonzero for the gapdependent capacitances of a sensor $X$-face domain, $\frac{\partial C_{S j}}{\partial x}$ vanishes for a typical sensor $Y$ or $Z$ domain far from the TM edge, as TM motion along $x$ gives fixed-gap sliding of a large conducting plane. With a patchwork TM surface, a sensor $Y$ or $Z$ domain "overlaps" with several opposing TM domains, giving $\frac{\partial C_{i j}}{\partial x} \neq 0$ and thus a force in the $x$ direction. The field component along the underlying conducting TM surface vanishes, but its gradient does not, creating a shear force on the TM surface dipole distribution that generates the varying surface potential [21, 31]. Stray torques from such shear forces limited sensitivity for the spherical Gravity Probe B gyroscopes [7].

Electrostatic shear is not essential to $\frac{\partial F_{x}}{\partial q}$ (see Ref. 32]); $\Delta_{x}$ reflects the average field along $x$ felt by the TM free charge, and the uniform change in $V_{T M}$ caused by $q$ does not create significant field gradients that shear the TM surface dipoles. However, shear forces impact attempts to measure $\Delta_{x}$ with applied voltages, and thus also the random charge problem.

Ideally, $\Delta_{x}$ is measured by the force caused by a change in TM charge, with voltages then applied to the $X$ electrodes to null $\frac{\partial F_{x}}{\partial q}$. An easier proposed method [25] simulates charge by modulating $V_{T M}$ with $V_{0} \sin 2 \pi f_{0} t$ applied to the $4 Z$ electrodes. Combining Eqns. 4 and 5 yields the coherent force

$$
\begin{array}{r}
F_{x(1 f)}=-V_{0} \sin 2 \pi f_{0} t \times \\
\left\{\alpha_{z}\left|\frac{\partial C_{X}}{\partial x}\right| \Delta_{x}-\sum_{i(T M), j\left(S_{z}\right)} \frac{\partial C_{i j}}{\partial x}\left(\delta V_{j}-\delta V_{i}\right)\right\}
\end{array}
$$

Here, $j\left(S_{z}\right)$ sums over domains on the modulated $Z$ electrodes and $\alpha_{z}=4 C_{Z} / C_{T} \approx 0.07$. The first term is proportional to $\Delta_{x}$, while the second, irrelevant to $\frac{\partial F_{x}}{\partial q}$, is the shear action of modulated field gradients near the $Z$ electrodes on nearby TM surface dipoles. It vanishes with an equipotential TM, with $\frac{\partial C_{S j}}{\partial x} \approx 0$ for a $\mathrm{Z}$ electrode domain $j$.

TM inclination with respect to the $Z$ electrodes also introduces error, with a gap-varying $\frac{\partial C_{Z}}{\partial x}$ coupling the $Z$-electrode surface potentials and modulation voltage. 


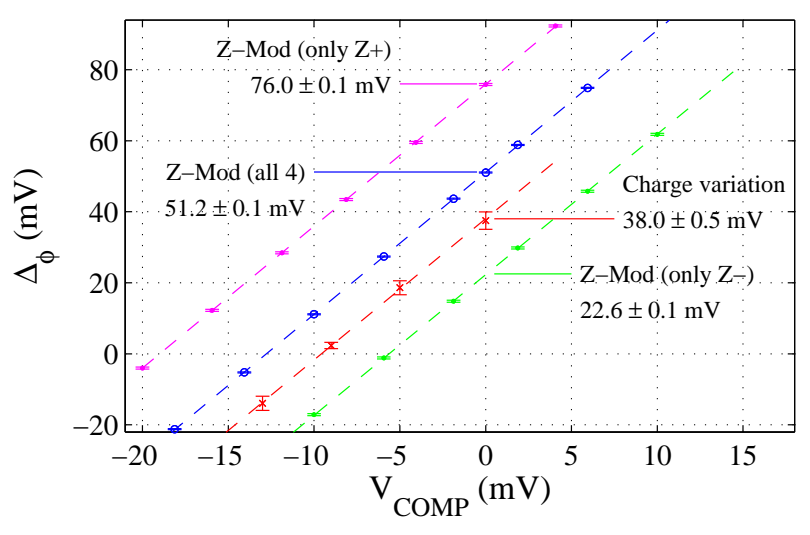

FIG. 3. Comparison of $\Delta_{\phi}$ obtained by charge variation and by the $Z$-modulation technique, with extracted values for the uncompensated $\Delta_{\phi}\left(V_{C O M P}=0\right)$. Single point error bars $(<300 \mu \mathrm{V})$ are not visible in the modulation data.

However, shear coupling to the varying TM potential represents a more fundamental error that limits any technique to measure $\frac{\partial F_{x}}{\partial q}$ with applied voltages - without actually varying $q$ - even with perfect alignment.

Experimentally, with a torsion pendulum sensitive to torque, $N_{\phi}$ (see Fig. 11), we assess $\Delta_{x}$ and its fluctuations by measuring the rotational imbalance $\Delta_{\phi}$ relevant to $\frac{\partial N_{\phi}}{\partial q} . \quad \Delta_{\phi}$ is defined analogously to $\Delta_{x}$ $(x \rightarrow \phi$ in Eqns. 1, 6, and 7). With an equipotential TM and individually equipotential electrodes, $\Delta_{x}$ and $\Delta_{\phi}$ become, respectively, the left-right and diagonal imbalances of the same $4 X$-electrode potentials, $\Delta_{x}=\left(V_{X 1+}+V_{X 2+}-V_{X 1-}-V_{X 2-}\right)$ and $\Delta_{\phi}=$ $\left(V_{X 1+}-V_{X 2+}-V_{X 1-}+V_{X 2-}\right)$ [25]. With electrostatically inhomogeneous conductors, $\Delta_{\phi}$ has gap-varying sensitivity to the $Y$ surface potentials as well as the $X$ domains that dominate $\Delta_{x}$ [32], and thus statistically overestimates $\Delta_{x}$ and its fluctuations.

The Z-modulation measurement of $\Delta_{\phi}$ is compared here with direct measurement of $\frac{\partial N_{\phi}}{\partial q}$ (see Fig. 3). Measurements are performed as a function of compensation voltage $V_{C O M P}$, applied with positive (negative) polarity on the $X 1+$ and $X 2-(X 1-$ and $X 2+)$ electrodes. We measure $\frac{\partial N_{\phi}}{\partial q}$ by the change in torque, up to $5 \mathrm{fNm}$ and measured to $\pm 0.1 \mathrm{fN} \mathrm{m}$, upon rapid change in TM charge, of order $10^{7} e$, caused by $10-30 \mathrm{~s}$ UV illuminations. The $Z$-modulation and charge measurement technique were applied and analyzed as in Refs. [16, 25].

The uncompensated $\Delta_{\phi} \approx 38 \mathrm{mV}$ measured by charge variation is in the typical range of other sensors [16, 17], and the slope of 4 confirms Eqns. 6.77 for 4 compensated $X$ electrodes. The result for 4 -electrode $Z$-modulation, $\Delta_{\phi} \approx 51 \mathrm{mV}$, is $13 \mathrm{mV}$ larger. Additional tests with modulation on only the $Z+$ or $Z$ - electrode pairs give values of $\Delta_{\phi}$ differing by more than $50 \mathrm{mV}$ (Fig. 3), with a $90 \mathrm{mV}$ range observed for $\Delta_{\phi}$ with individual modula- tion of the $4 Z$ electrodes.

The disagreement of the various $Z$-modulation results with $\Delta_{\phi}$ measured with $\frac{\partial N_{\phi}}{\partial q}$ indicates the level of error in the $Z$-modulation technique. With an equipotential TM, these measurements should yield the same value. The variation between results with different $Z$ electrodes reflects differences in the TM potentials near the different $Z$-electrodes. This partially averages out by modulating all $4 Z$-electrodes, but still leaves a $13 \mathrm{mV}$ deviation from the true $\Delta_{\phi}$. For comparison and an indication of long term stability, the same sensor 1 year before gave $\Delta_{\phi} \approx$ $135 \mathrm{mV}$, with an $8 \mathrm{mV}$ difference between the $\frac{\partial N_{\phi}}{\partial q}$ and $Z$-modulation techniques.

To limit the random charge contribution to the LISA acceleration noise (Fig. 6), we want $\Delta_{x}<10 \mathrm{mV}$. This will likely require in-flight measurement and compensation of intrinsic imbalances typically of order $100 \mathrm{mV}$, repeated periodically, given the slow drifts observed here and elsewhere [18]. Additionally, analysis and measurements indicate that errors associated with the $Z$ modulation technique may not allow $10 \mathrm{mV}$ accuracy. This would require the more cumbersome direct measurement of $\frac{\partial F_{x}}{\partial q}$ in flight, which needs UV light actuation, charge measurements, and transient force detection.

Noise in $\Delta_{x}$ is assessed with two different measurements of $S_{\Delta_{\phi}}$. We first measure torque noise with a charged TM, attributing any excess to $\Delta_{\phi}$ fluctuations,

$$
S_{N}(q)-S_{N}(0)=\left[\frac{q}{C_{T}}\left|\frac{\partial C_{X}}{\partial \phi}\right|\right]^{2} S_{\Delta_{\phi}} .
$$

Measurements for 3 consecutive weekends with the TM charged to $V_{T M}=1.82 \pm 0.02 \mathrm{~V}\left(q \approx 4 \times 10^{8} e\right)$ were sandwiched between 4 weekends with the TM neutral to within $20 \mathrm{mV}$. The noise analysis, similar to Ref. [33], uses $25000 \mathrm{~s}$ Blackman-Harris windows with $66 \%$ overlap - 55 and 70 windows for, respectively, the charged and neutral TM data - binned into 8 frequencies per decade and averaged, with uncertainties based on standard deviation among pre-averaged groups of 5 windows.

Figure 4 shows averaged torque noise, similar for the charged and neutral TM and with a minimum near $3 \mathrm{mHz}$ of roughly $0.7 \mathrm{fNm} / \mathrm{Hz}^{1 / 2}\left(120 \mu \mathrm{V} / \mathrm{Hz}^{1 / 2}\right)$. Following Eqn. 8, we subtract the neutral TM background $S_{N}(0)-$ measured to be stationary at the $0.1(\mathrm{fN} \mathrm{m})^{2} / \mathrm{Hz}$ level to obtain $S_{\Delta_{\phi}}$ in Fig. 5. The $1-4 \mathrm{mHz}$ average is roughly $50 \mu \mathrm{V} / \mathrm{Hz}^{1 / 2}$, resolved at nearly the $2 \sigma$ level. Background noise, and the associated errors bars, increase at both higher and lower frequencies, with no resolvable excess.

We also measure residual fluctuations in the $Z$ modulation signal, as in Ref. [17] and Eqn. 7], with $\Delta_{\phi}(t)$ detected in the coherent torque amplitude at the modulation frequency $f_{0}$. This overestimates $S_{\Delta_{\phi}}$ by the second term in Eqn. 7] but improves sensitivity to low frequency fluctuations in $\Delta_{\phi}$, which are spectrally upshifted to amplitude modulate the torque carrier around 


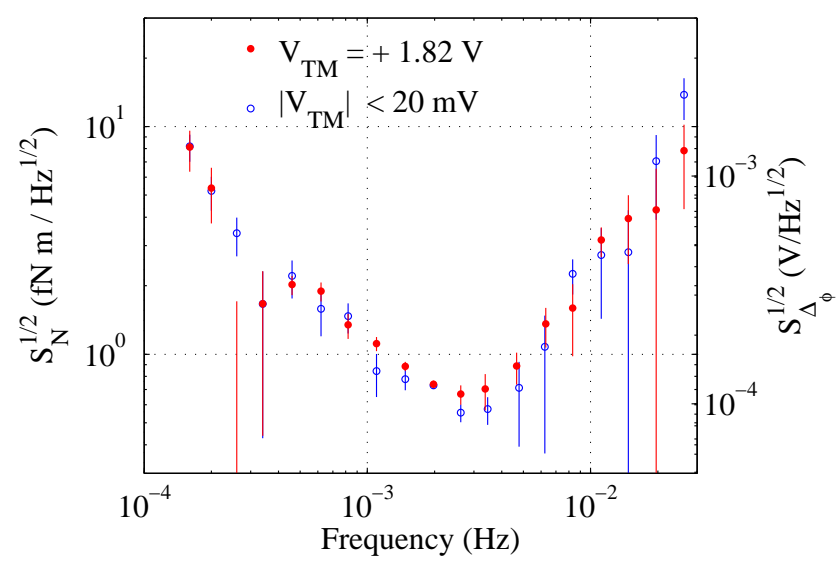

FIG. 4. Pendulum torque noise measured with the TM nearly neutral and when charged to a potential $V_{T M}=+1.82$ $\mathrm{V}$, with conversion into $S_{\Delta_{\phi}}$ shown at right.

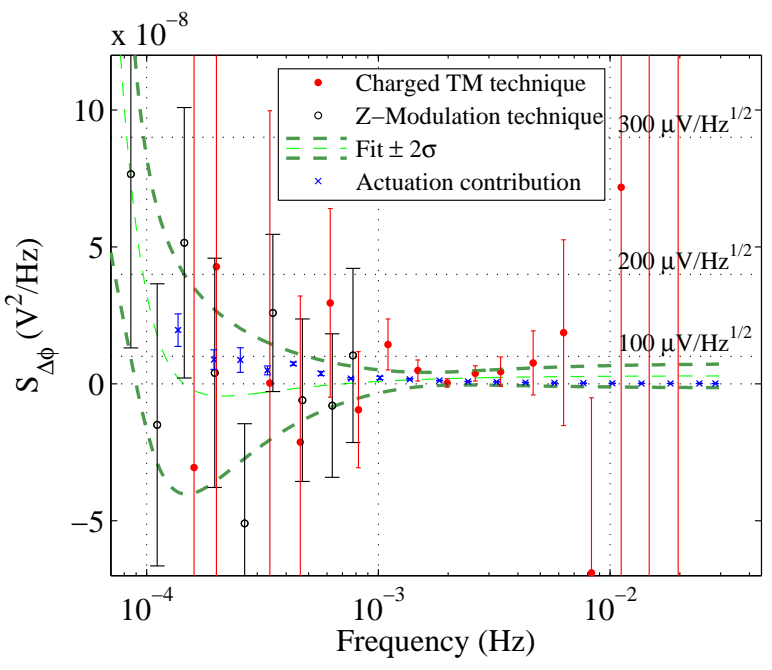

FIG. 5. Stray potential noise $S_{\Delta_{\phi}}$, measured with two techniques. Statistically insignificant large error points are omitted from each dataset. Also shown are a fit to the joint dataset and the noise contribution from the actuation electronics.

$f_{0}=3 \mathrm{mHz}$, chosen to minimize torque noise. $V_{C O M P}$ is adjusted to null the signal upon starting the measurement. $\Delta_{\phi}(t)$ is corrected for the measured dependence on tilt-induced TM translation inside the sensor. We subtract background measurement noise, typically $500 \mu \mathrm{V} / \mathrm{Hz}^{1 / 2}$ as calculated with the demodulated quadrature (cosine) torque phase, which contains statistical torque noise without electrostatic signal. Spectra are calculated with $60000 \mathrm{~s}$ windows, binned and averaged, and then background subtracted for each of 13 weekend measurements, with uncertainties based on scatter between different windows. Figure 5 shows a weighted mean of these data.

The measurements with a charged TM $(\bullet)$ and Zmodulation (o) combine in Fig. 50 [34] for a significant upper limit on the noise power $S_{\Delta_{\phi}}$ from 0.1 to $5 \mathrm{mHz}$.
We fit the combined dataset to various models with a low frequency increase - Fig. 5 shows a fit with $f^{-1}$ and $f^{-4}$ terms - and find $2 \sigma$ confidence intervals of $(0,80) \mu \mathrm{V} / \mathrm{Hz}^{1 / 2}$ at $1 \mathrm{mHz}$ and $(0,290) \mu \mathrm{V} / \mathrm{Hz}^{1 / 2}$ at $0.1 \mathrm{mHz}$. Also shown $(\times)$ is the contribution from the 4 $X$-electrode actuation circuits [35], measured separately to give $f^{-1}$ noise power with roughly $50 \mu \mathrm{V} / \mathrm{Hz}^{1 / 2}$ at $1 \mathrm{mHz}$. The weakly detected fluctuations are thus consistent with electronic noise, and we do not resolve true surface potential fluctuations in the LISA bandwidth. $\Delta_{\phi}$ effectively sums the noise of many $(\approx 16$, see Ref. 32 ]) areas the size of a LISA $X$-electrode $\left(\approx 500 \mathrm{~mm}^{2}\right)$, and, for fluctuations that are uncorrelated on larger spatial scales, our $2 \sigma$ upper limit corresponds to $12 \mu \mathrm{V} / \mathrm{Hz}^{1 / 2}$ in the average potential difference between $500 \mathrm{~mm}^{2}$ surface regions.

The $+2 \sigma$ curve in Fig. 5 is taken as a upper limit for $S_{\Delta_{x}}$ in the acceleration noise budget in Fig. 6. Assuming a TM charge of $10^{7} e$, this result is compatible with the LISA goals, marginally so at $0.1 \mathrm{mHz}$. This significantly improves upon previous upper limits with LISA prototype hardware, from roughly $1 \mathrm{mV} / \mathrm{Hz}^{1 / 2}$ [17] to $80 \mu \mathrm{V} / \mathrm{Hz}^{1 / 2}$ at $1 \mathrm{mHz}$. The limit is also below the $150-200 \mu \mathrm{V} / \mathrm{Hz}^{1 / 2}$ deduced for $S_{\Delta_{x}}^{1 / 2}$ at $1 \mathrm{mHz}$ from observations of potential fluctuations between opposing goldcoated plates [18, 32].

Several design aspects merit consideration for improving upon the noise budget in Fig. 6] A thin ground wire can eliminate TM charge [4, 36], but introduces thermal mechanical noise well beyond the $\mathrm{fm} / \mathrm{s}^{2} / \mathrm{Hz}^{1 / 2}$ level [37]. With a floating TM, the $q-\delta V$ interaction has roughly $d^{-1}$ dependence (Eqn. 11). Larger gaps help, but even a factor $10, d=4 \mathrm{~cm}$, will lower the random charge noise in Fig. 6 only if $\Delta_{x}<100 \mathrm{mV}$, which may still require voltagecontrolled electrodes for compensation. This introduces actuation circuitry noise, which sums with, or even dominates over, surface potential fluctuations. Preliminary measurements for the LISA Pathfinder electronics indicate a circuit contribution $S_{\Delta_{x}}^{1 / 2} \approx 30 \mu \mathrm{V} / \mathrm{Hz}^{1 / 2}[38]$.

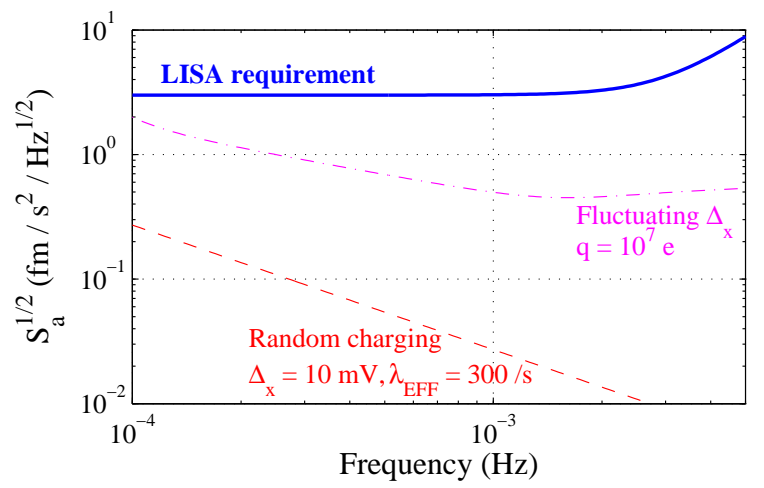

FIG. 6. Conservative acceleration noise budget for the $q-\delta V$ interaction, with (bold) the LISA goal. 
Along with the interaction with TM charge, stray potential fluctuations can also create force noise by mixing with stable domain potentials, even with $q=0$, as allowed by Eqn. [5. Analysis of this effect demands combining averaged potential fluctuation data with the domain spatial distribution and correlations. This will impact in-flight operation issues such as the extent to which continuous TM discharge can reduce noise.

This work was supported by the Istituto Nazionale di Fisica Nucleare, the Agenzia Spaziale Italiana (LISA Pathfinder contract), and the Italian Ministry of University and Research (PRIN 2008).

\section{Supplemental material for The interaction between stray electrostatic fields and a charged free-falling test mass: \\ Statistical comparison of different stray potential measurements and their application to the interaction with TM charge}

This supplementary report addresses how - and which - stray electrostatic potentials create a force on a charged test mass (TM),

$$
\frac{\partial F_{x}}{\partial q} \equiv-\frac{1}{C_{T}} \frac{\partial C_{X}}{\partial x} \Delta_{x}
$$

and how to compare different stray potential measurements with $\Delta_{x}$, and its fluctuations, for the LISA geometry. All prototype sensors or sample surfaces will be different, even with nominally identical gold surfaces prepared in the same way. As such, we seek a statistical comparison between different measurements under various assumptions for the underlying distribution of stray potentials. The figure of merit that we will use is the mean square variance $\left\langle\Delta_{x}^{2}\right\rangle$ of the potential difference relevant to charging in LISA. The noise power spectral density should scale in the same fashion, as it is the Fourier transform of the related correlation, $\left\langle\Delta_{x}(t) \Delta_{x}(t+\tau)\right\rangle$.

The principle conclusions of this report are:

- The coupling to charge $\frac{\partial F_{x}}{\partial q}$ is essentially an average electrostatic field along $x$. It (and equivalently $\Delta_{x}$ ) is thus dominated by the stray potentials on the surfaces of the TM and surrounding enclosure that are normal to the $x$ axis.

- The rotational stray potential imbalance $\Delta_{\phi}$, studied experimentally in the main article, is statistically noisier than $\Delta_{x}$ for stray potential distributions that are dominated by domains the size of the LISA electrodes $\left(500 \mathrm{~mm}^{2}\right)$ or smaller.

- Average potential difference measurements performed in the geometry of Ref. [18] are readily applicable to the LISA geometry. To compare with $\Delta_{x}$, which is normalized to the size of a single LISA $X$ electrode, those results (for linear spectral noise density) must be multiplied by a factor 5 - 6 for any characteristic domain sizes up to the dimensions $(\approx$ $2000 \mathrm{~mm}^{2}$ ) of the surfaces measured.

In the capacitive model, the potential difference $\Delta_{x}$,

$$
\Delta_{x}=\frac{1}{\left|\frac{\partial C_{X}}{\partial x}\right|} \sum_{i(T M), j(S)} \frac{\partial C_{i j}}{\partial x}\left(V_{j}-\delta V_{i}\right)
$$

can also be expressed

$$
\Delta_{x}=\frac{1}{\left|\frac{\partial C_{X}}{\partial x}\right|}\left[\sum_{j(S)} \frac{\partial C_{S j}}{\partial x} V_{j}-\sum_{i(T M)} \frac{\partial C_{T M} i}{\partial x} \delta V_{i}\right]
$$

where $C_{S j} \equiv \sum_{i(T M)} C_{i j}$ is the total capacitance between sensor domain $j$ and the entire TM, and $C_{T M i} \equiv \sum_{j(S)} C_{i j}$ is the total capacitance between TM domain $i$ and the surrounding sensor. $\frac{\partial C_{S j}}{\partial x}$ and $\frac{\partial C_{T M i}}{\partial x}$ are positive for domains on the sensor or TM $X+$ faces and negative for domains on the $X$ - faces, as the relevant gaps change with TM motion along $x$. The same derivatives are nearly zero for domains on the $Y$ or $Z$ faces; TM slide motion along $x$ changes the individual inter-domain capacitances by altering their effective overlap (see Fig. 7), but the total capacitance of a domain to the opposing surface is unchanged. This is no longer true near the TM edge, but border effects play a secondary role for an enclosure with TM size much larger than the relevant TM - sensor gaps, as is the case for LISA, with cube sidelength $s=46 \mathrm{~mm}$ and gap $d=4 \mathrm{~mm}$. Aside from the normalization factor $\frac{\partial C_{X}}{\partial x}$, the two terms in Eqn. 11 represent weighted averages of the domain potentials on the sensor and TM $X$ faces, with the weights provided by the capacitance derivatives, which are proportional to area for uniform $d$. These average potentials determine the average residual electrostatic field along $x$, and thus also $\Delta_{x}$.

With $\Delta_{x}$ dominated by the average potential differences between opposing TM and sensor surfaces, we construct an approximate statistical model, which considers the average difference $\delta V_{m}$ between opposing TM and sensor surface elements of area $\Delta a$ (see Fig. 8). Such elements are not necessarily equipotential but are, first, large enough $\left(\Delta a>d^{2}\right)$ to allow, for ease of calculation, an infinite parallel plate model for the capacitance derivatives, $\frac{\partial C_{m}}{\partial x} \approx \frac{\Delta a \epsilon_{0}}{d^{2}}$, such that

$$
\Delta_{x} \approx \frac{d^{2}}{A_{x} \epsilon_{0}} \sum_{m} \frac{\Delta a \epsilon_{0}}{d^{2}} \delta V_{m}=\frac{\Delta a}{A_{x}} \sum_{m} \delta V_{m}
$$

where $\frac{\partial C_{X}}{\partial x} \approx \frac{A_{x} \epsilon_{0}}{d^{2}}$. Second, $\Delta a$ is considered large enough such that the average potential is uncorrelated between elements, with $\left\langle\delta V_{m} \delta V_{n}\right\rangle=\delta_{m, n} V_{0}^{2}$, where $V_{0}^{2}$ is the mean square value of the potential difference averaged over surface $\Delta a$. As such, we can estimate the 
statistical variance $\left\langle\Delta_{x}^{2}\right\rangle$

$$
\left\langle\Delta_{x}^{2}\right\rangle \approx \frac{\Delta a^{2}}{A_{x}^{2}} \sum_{m, n}\left\langle\delta V_{m} \delta V_{n}\right\rangle=\frac{\Delta a^{2}}{A_{x}^{2}} V_{0}^{2} N=\Delta a V_{0}^{2} \frac{2 s^{2}}{A_{x}^{2}}
$$

with $N \approx \frac{2 s^{2}}{\Delta a}$ the number of elements on the two $X$ faces. We will confront this variance with other potential difference measurements under different assumptions of the minimum area $\Delta a$ for which surface elements can be considered uncorrelated.

The same arguments apply for the rotational imbalance $\Delta_{\phi}$, defined

$$
\Delta_{\phi}=\frac{1}{\left|\frac{\partial C_{X}}{\partial \phi}\right|} \sum_{i(T M), j(S)} \frac{\partial C_{i j}}{\partial \phi}\left(V_{j}-\delta V_{i}\right)
$$

We can perform a similar analysis, using an infinite wedge approximation to estimate the gap-dependent capacitance derivatives, with $\frac{\partial C_{m}}{\partial \phi} \approx \frac{\Delta a \epsilon_{0}}{d^{2}} l_{m}$ for an element of area $\Delta a$ at a distance $l_{m}$ from the center of the TM face (see Fig. 8). Likewise, $\Delta_{\phi}$ normalizes to the $X$ electrode derivative $\frac{\partial C_{X}}{\partial \phi} \approx \frac{A_{x} \epsilon_{0} R_{x}}{d^{2}}$. Both $X$ and $Y$ faces give gap-dependent contributions to $\Delta_{\phi}$,

$$
\begin{aligned}
\Delta_{\phi} & \approx \frac{d^{2}}{A_{x} R_{x} \epsilon_{0}} \sum_{m} \frac{\Delta a l_{m} \epsilon_{0}}{d^{2}} \delta V_{m}=\frac{\Delta a}{A_{x} R_{x}} \sum_{m} l_{m} \delta V_{m} \\
\left\langle\Delta_{\phi}^{2}\right\rangle & \approx \Delta a V_{0}^{2} \frac{4 s^{2} \bar{l}^{2}}{A_{x}^{2} R_{x}^{2}}
\end{aligned}
$$

Here, $N=\frac{4 s^{2}}{\Delta a}$ for the $4 X / Y$ faces and $\overline{l^{2}}$ is a mean square armlength, with $\overline{l^{2}}=\frac{2}{s} \int_{0}^{s / 2} x^{2} d x=\frac{s^{2}}{12}$ for $\Delta a \ll s^{2}$. This is approximate in the case of only a

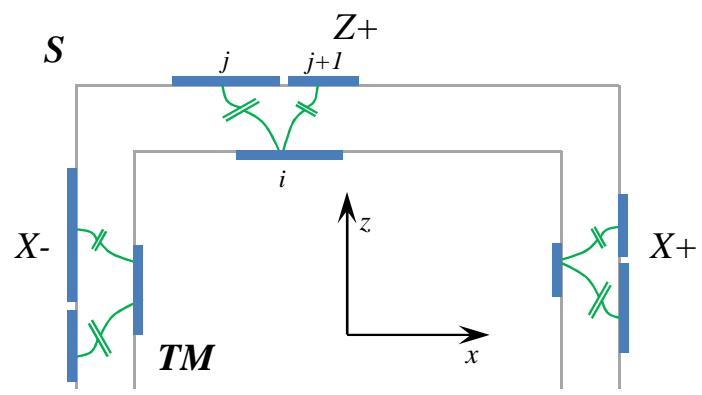

FIG. 7. Cartoon illustrating the capacitive coupling between domains on the TM and surrounding enclosure (or sensor, $S$ ). Adjacent elements on the $X$ sensor faces contribute $\frac{\partial C_{m, n}}{\partial x}$ with the same sign, positive on the $X+$ face and negative on $X-$, as they undergo the same gap change upon TM motion along $x$. The $x$ dependence of the adjacent domains on a $Z$ face cancel out to first approximation, as the decrease in area overlap between TM domain $i$ and sensor domain $j$ is matched by the decrease in the $(i, j+1)$ overlap, resulting in $\frac{\partial C_{T M i}}{\partial x} \approx 0$. Ultimately, this gives a weak dependence of the charge coupling $\frac{\partial F_{x}}{\partial q}$ (or $\Delta_{x}$ ) on the $Y$ and $Z$-face surface potentials.

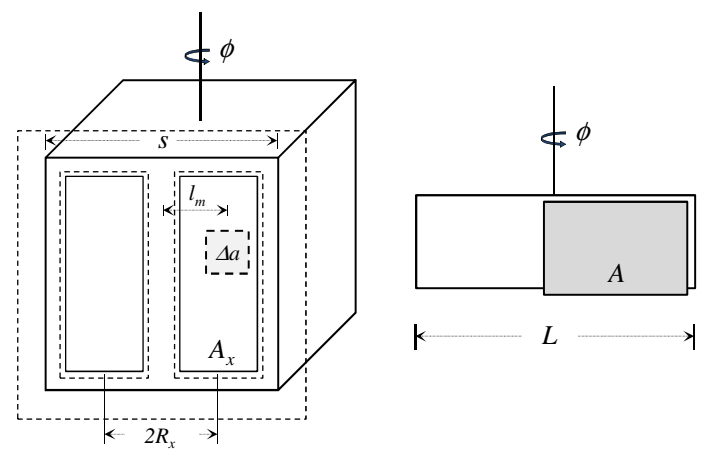

FIG. 8. Schematic illustration of two experimental configurations for torque measurements of surface potential differences. At left is a LISA prototype TM (cube sidelength $s=46 \mathrm{~mm}$ ), with the outlines of the sensor $X$ electrode footprints, with surface area $A_{x}=529 \mathrm{~mm}^{2}$ and a on-center semi-separation $R_{x}=10.75 \mathrm{~mm}$ and (dashed) guard ring surfaces. The gap $d_{x}$ from TM to the $X$ electrodes and adjacent grounded sensor guard ring surfaces is $4 \mathrm{~mm}$. At right is the geometry of Ref. [18], in which an average potential difference is measured between one half of the suspended plate and a facing electrode plate of width $\frac{L}{2}=57.2 \mathrm{~mm}$ and area $A=2180 \mathrm{~mm}^{2}$.

few uncorrelated domains per TM face, such as for $\Delta a$ the size of an $X$ electrode $\left(\approx 500 \mathrm{~mm}^{2}\right)$.

Table [1 summarizes the statistical variation for $\Delta_{\phi}$ compared to that of $\Delta_{x}$ for different assumptions of the relevant minimum area $\Delta a$ beyond which the average surface potentials become uncorrelated. In the limit of small correlation-length domains $\left(\Delta a \ll s^{2}\right)$, the variance $\left\langle\Delta_{\phi}^{2}\right\rangle \approx 3\left\langle\Delta_{x}^{2}\right\rangle$, with a factor 2 from doubling, from 2 to 4 , the faces with gap-dependent contributions to $\Delta_{\phi}$ and a factor $\frac{s^{2}}{12 R_{x}^{2}} \approx 1.5$ for the average square armlength compared to that of a single $X$ electrode.

If, instead, all distinct conducting surfaces - for LISA, the TM, the individual sensor electrodes, and the rest of the electrode housing - were individually equipotential, then $\Delta_{x}$ and $\Delta_{\phi}$ are both determined only by the stray potential values on the $4 X$ electrodes, as these are the only full conductors with a non-vanishing $\frac{\partial C}{\partial x}$ and $\frac{\partial C}{\partial \phi}$. $\Delta_{x}$ and $\Delta_{\phi}$ are thus different combinations of these 4 potentials in this case, as in the simplified analysis of Ref. 25], and their expected statistical variances are equal.

In the case that each entire sensor and TM face is a distinct equipotential, then $\Delta_{\phi}=0$, as the contribution of one half of a sensor (or TM) face cancels that of the other half, with $\frac{\partial C}{\partial \phi}$ changing sign. $\Delta_{x}$ could still be nonzero in this case, and thus measurement of $\Delta_{\phi}$ would no longer be a good indicator of the statistics of $\Delta_{x}$. This case is, however, considered highly unlikely in the case of the LISA prototype sensor under study, where the individual faces of the sensor, including electrodes and surrounding guard ring surfaces, are composed of physically separate conductors, whose gold coatings are connected eletrically only through the attached circuitry. 
As such, the distribution of $\Delta_{\phi}$, and its fluctuations, are taken as a statistical indicator for $\Delta_{x}$ that, considering Table】 is slightly pessimistic over a range of assumptions for the underlying potential distribution.

It is interesting to scale the measured values for the noise $S_{\Delta_{\phi}}$ to the corresponding noise in the average potential on a conductor of given size, for instance that of a single LISA $X$ electrode. Substituting $\Delta a=A_{x}$ in Eqn. [15] and approximating $\overline{l^{2}} \approx \frac{s^{2}}{12}$, we find $\left\langle\Delta_{\phi}^{2}\right\rangle \approx$ $V_{0}^{2} \frac{s^{4}}{3 A_{x} R_{x}^{2}} \approx 25 V_{0}^{2}$ (corresponding to a relevant surface area $16 \times A_{x}$ on the $4 X$ and $Y$ faces and the factor 1.5 armlength correction mentioned above). As such, in the limit that the potential fluctuations are correlated only on a scale smaller than $A_{x} \approx 500 \mathrm{~mm}^{2}$, the measured noise in $\Delta_{\phi}$ is roughly 5 times larger, in linear spectral density, than the noise in the average potential difference between opposing $500 \mathrm{~mm}^{2}$ surfaces.

In the geometry studied in Ref. [18], shown at right in Fig. 8, a modulated voltage is applied between two parallel plates, with relevant overlap width $\frac{L}{2}$ and height $h$ (and thus area $A=\frac{h L}{2}$ ). The measured torque is converted into an equivalent potential difference between the two plates by dividing by the total capacitive derivative $\frac{\partial C}{\partial \phi}$. Given this normalization and the electrostatic model used in our text, the measured potential difference in these measurements, $V_{U W}$, is given by

$$
V_{U W}=\frac{1}{\left|\frac{\partial C}{\partial \phi}\right|} \sum_{i(1), j(2)} \frac{\partial C_{i j}}{\partial \phi}\left(\delta V_{j}-\delta V_{i}\right)
$$

Following the same analysis applied for $\Delta_{x}$ and $\Delta_{\phi}$,

\begin{tabular}{|c|c|c|c|}
\hline \hline & $\begin{array}{c}\Delta a \ll s^{2} \\
\left(\text { or } \Delta a \approx A_{x}\right)\end{array}$ & $\Delta a \approx s^{2}$ & $\begin{array}{r}\text { equipotential } \\
\text { conductors }\end{array}$ \\
\hline$\frac{\left\langle\Delta_{\phi}^{2}\right\rangle}{\left\langle\Delta_{x}^{2}\right\rangle}$ & $\frac{s^{2}}{6 R_{x}^{2}} \approx 3$ & --- & 1 \\
\hline$\frac{\left\langle V_{U W}^{2}\right\rangle}{\left\langle\Delta_{x}^{2}\right\rangle}$ & $\frac{2 A_{x}^{2}}{3 A s^{2}} \approx 0.04$ & $\frac{A_{x}^{2}}{2 s^{4}} \approx 0.03$ & $\approx 0.03$ \\
\hline \hline
\end{tabular}

TABLE I. Values for scaling potential differences in torque measurements of $\Delta_{\phi}$ and $V_{U W}$ to the $\Delta_{x}$ relevant to the interaction with TM charge in LISA. We consider cases in which the minimum surface area of the underlying characteristic domains, beyond which potentials become uncorrelated, are (1) smaller than the TM cube sidelength $s,(2)$ roughly equal to the TM dimension $s$, and (3) coincide with the individual conducting surface boundaries - TM and electrodes for LISA and the individual plates in the configuration of Ref. [18] so that each has a single uniform potential. As discussed in the text, $\Delta_{\phi}$ is not a statistical indicator of $\Delta_{x}$ in the case $\left(\Delta a \approx s^{2}\right)$ that each TM and sensor face is a unique equipotential. Cases 2 and 3 coincide for $V_{U W}$, in which the only two relevant conductors have $A \approx s^{2}$. with $\frac{\partial C}{\partial \phi} \approx \frac{A \epsilon_{0} \frac{L}{4}}{d^{2}}$

$$
\begin{aligned}
V_{U W} & \approx \frac{\Delta a}{A \frac{L}{4}} \sum_{m} l_{m} V_{m} \\
\left\langle V_{U W}^{2}\right\rangle & \approx \Delta a V_{0}^{2} \frac{16 \bar{l}^{2}}{A L^{2}}
\end{aligned}
$$

The surface area $A \approx 2180 \mathrm{~mm}^{2}$ used in the measurement - and in the normalization of $V_{U W}$ - is roughly that of a LISA TM face, $s^{2}$, and four times that of a LISA sensor $X$ electrode. In rough terms, the mean square variance in $V_{U W}$ will be smaller than that of $\Delta_{x}$ by a factor 16 - due to the factor 4 in normalization area, $\frac{A}{A_{x}}$ (see Eqns. 10, 12 and 16]17) - and by an additional statistical factor 2 , for the ratio of relevant surface area, $\frac{2 s^{2}}{A}$, which means half the number of domains in the UW geometry, regardless of their size. Including a small armlength correction factor weighing domains farther from the torque axis, which varies from 1 for $\Delta a \approx s^{2}$ to $\frac{4}{3}$ for $\Delta a \ll s^{2}$, the standard deviation in $V_{U W}^{2}$ is $25-35$

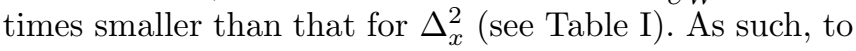
deduce an expectation value for the noise in $\Delta_{x}$ based on potential fluctuation measurements in the UW geometry, we must scale the linear noise density by a factor $5-6$.

[1] D. Ugolini, R. McKinney, and G. M. Harry, Rev. of Sci. Instr. 78, 046102 (2007).

[2] M. Hewitson et al., Class. Quantum Grav. 24, 6379 (2007).

[3] P. Bender et al, LISA ESA-SCI(2000)11, 2000.

[4] R. Chhun et al., Acta Astronautica 60, 873 (2007).

[5] D. J. Kapner et al., Phys. Rev. Lett. 98, 021101 (2007).

[6] J. C. Long et al., Nature (London) 421, 922 (2003).

[7] C. W. F. Everitt et al., Phys. Rev. Lett. 106, 221101 (2011).

[8] S. Buchman and J. Turneaure, Rev. Sci. Inst. 82, 074502 (2011).

[9] M. Armano et al., Class. Quantum Grav. 26, 094001 (2009).

[10] F. Antonucci et al., Class. Quantum Grav. 28, 094002 (2011).

[11] R. Dolesi et al., Class. Quantum Grav. 20, S99 (2003).

[12] W. J. Weber et al., SPIE Proc. 4856, 31 (2002).

[13] H. M. Araùjo et al., Astroparticle Physics 22, 451 (2005).

[14] J. B. Camp, T. W. Darling, and R. E. Brown, J. Appl. Phys. 69, 7126 (1991).

[15] C. C. Speake and C. Trenkel, Phys. Rev. Lett. 90, 160403 (2003).

[16] L. Carbone et al., Phys. Rev. Lett. 91, 151101 (2003).

[17] L. Carbone et al., Class. Quantum Grav. 22, S509 (2005).

[18] S. E. Pollack, S. Schlamminger, and J. H. Gundlach, Phys. Rev. Lett. 101, 071101 (2008).

[19] N. A. Robertson et al., Class. Quantum Grav. 23, 2665 (2006).

[20] D. A. S. Shaul et al., Class. Quantum Grav. 22, S297 (2005).

[21] C. C. Speake, Class. Quantum Grav. 13, A291 (1996). 
[22] B. Schumaker, Class. Quantum Grav. 20, S239 (2003).

[23] R. T. Stebbins et al., Class. Quantum Grav. 21, S653 (2004).

[24] P. L. Bender, Class. Quantum Grav. 20, S305 (2003).

[25] W. J. Weber et al., Advances in Space Research 39, 213 (2007).

[26] A. Cavalleri et al., Class. Quantum Grav. 26, 094012 (2009).

[27] H. Vocca et al., Class. Quantum Grav. 21, S665 (2004).

[28] This is equivalent to the capacitive matrix formulation, with $q_{i}=\sum_{j} c_{i j} V_{j}$ and energy $U=\frac{1}{2} \sum_{i, j} c_{i j} V_{i} V_{j}$. The symmetries imposed by the Laplace equation [39] allow the electrical circuit analogy, with $c_{i j}=c_{j i}=-C_{i j}$ for $j \neq i$ and $c_{i i}=\sum_{j \neq i} C_{i j}$. From this Eqn. 5 follows.

[29] We choose the convention $\sum_{i(T M), j(S)} C_{i j} \delta V_{i}=0$, such that $V_{T M}=0$ when $q=0$ and all sensor domains are grounded, $V_{S j}=0$.

[30] N. Brandt and W. Fichter, J. Phys.: Conf. Ser. 154, 012008 (2009).

[31] J. D. Jackson, Classical Electrodynamics (Wiley, New York, 1975).

[32] See supplementary material in the appendix for a dis- cussion of the stray potentials that dominate $\Delta_{x}$ and an approximate model for comparing stray potential measurements in different geometries.

[33] A. Cavalleri et al., Class. Quantum Grav. 26, 094017 (2009).

[34] For both measurements, the estimated noise power excess $S_{\Delta_{\phi}}$ is in many cases smaller than the measurement uncertainty, and so negative data points are statistically inevitable. These negative data do not allow a conventional log-scale plot of linear spectral density $S_{\Delta_{\phi}}^{1 / 2}$ and force a linear plot of the power spectral density. Key linear spectral levels like $100 \mu \mathrm{V} / \mathrm{Hz}^{1 / 2}$ are shown as a guide.

[35] The actuation noise, measured with a chopperdemodulation technique, comes from commercial digitalto-analog converter (NI-6703) and instrumentation amplifier (AD-622) components.

[36] P. Touboul et al., Aerospace Sci. Technol. 8, 431 (2004).

[37] E. Willemot and P. Touboul, Rev. of Sci. Instr. 71, 302 (2000).

[38] C. Praplan, Tech. Rep. S2-HEV-RP3042, HEV-SO Valais, LISA Pathfinder (2009).

[39] W. J. Herrera and R. A. Diaz, Am. J. Phys. 76, 55 (2008). 